\title{
Condensed anisotropic diffusion for speckle reducton and enhancement in ultrasonography
}

\author{
Kalaivani Shanmugam ${ }^{*}$ and Wahidabanu RSD ${ }^{2}$
}

\begin{abstract}
This article proposes a technique for speckle reduction in medical ultrasound (US) imaging which preserves the point and linear features with the added advantage of energy condensation regulator. Whatever be the post processing task on US image, the image should undergo a preprocessing step called despeckling. Nowadays, though the US machines are available with built-in speckle reduction facility, they are suffered by many practical limitations such as limited dynamic range of the display, limited number of unique directions that an US beam scan follow to average an image and limited size of transducer, etc. The proposed diffusion model can be used as a visual enhancement tool for interpretation as well as a preprocessing task for further diagnosis. This method incorporates two terms: diffusion and regulator. The anisotropic diffusion preserves and enhances edges and local details. The regularization enables the correction of feature broadening distortion which is the common problem in second-order diffusion-based methods. In this scheme, the diffusion matrix is designed using local coordinate transformation and the feature broadening correction term is derived from energy function. Performance of the proposed method has been illustrated using synthetic and real US data. Experiments indicate better speckle reduction and effective preservation of edges and local details.
\end{abstract}

Keywords: Speckle, Filtering, Anisotropic diffusion, Regulator, Edge enhancement

\section{Introduction}

For more than two decades, ultrasonography has been considered as one of the most powerful techniques for imaging organs and soft tissue structures in the human body. Today, it is being used at an ever-increasing rate in the field of medical diagnostic technology. Ultrasonography is often preferred over other medical imaging modalities because it is non-invasive, portable, versatile, does not use ionizing radiations, and also relatively of low-cost. The images produced by commercial ultrasound (US) systems are usually optimized for visual interpretation because of its real-time usage. However, the usefulness of medical ultrasonography is degraded by signal-dependent noise called 'speckle' which is multiplicative in nature.

Imaging speckle is a phenomenon that occurs when a coherent source and a non-coherent detector are used to interrogate a medium, which is rough on the scale of

\footnotetext{
*Correspondence: s_kalai@rediffmail.com

'Department of Electronics and Communication Engineering, TRP Engineering College (SRM Group), Irungalur, Trichirappalli, Tamilnadu, India-621105

Full list of author information is available at the end of the article
}

the wavelength. In medical images, noise suppression is particularly a delicate and difficult task. A tradeoff between noise reduction and the preservation of actual image features has to be made in a way that enhances the diagnostically relevant image content. Speckle reduction is needed for two main reasons: first is to improve the human interpretation, i.e., for visual enhancement and secondly, despeckling is the preprocessing step for many image processing tasks. Importance of despeckling is depicted in Figure 1.

Many filters have been developed to cope up with speckle, with differences lying in the assumptions about the speckle model [1]. The methods described by Lee [2], Frost et al. [3], and Kuan et al. [4,5] are based on multiplicative model and simple logarithmic operation converts the speckle into additive noise. Filtering based on anisotropic diffusion (AD) was introduced by Perona and Malik [6] who had constituted a powerful tool for signal and image enhancement. When $\mathrm{AD}$ is introduced for first time an undesirable effect called "pin hole effect" may result and this is addressed by Monteil and Beghdadi [7] through optical flow technique. Later, Yu and Acton 


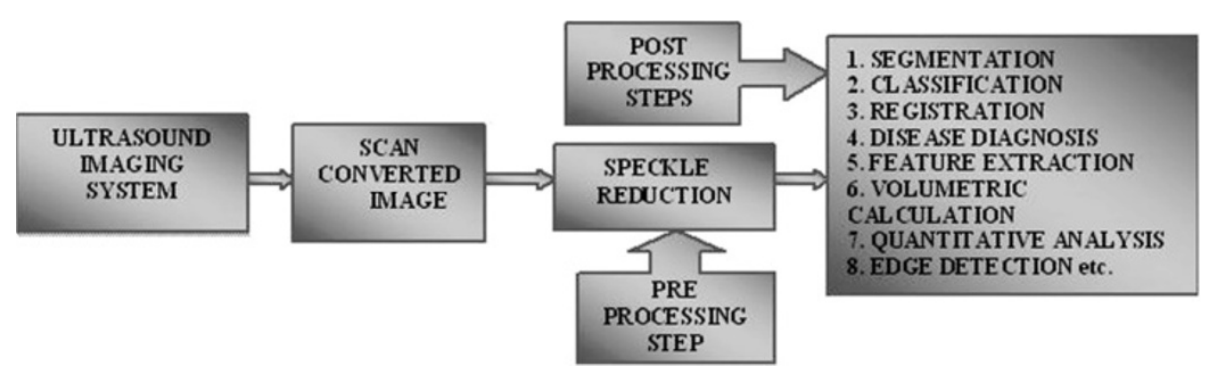

Figure 1 Importance of despeckling.

[8] have proposed a novel filtering scheme based on the filters first described by Lee and Frost. The authors find a relation between the former and the $\mathrm{AD}$ equation and give rise to a speckle removal filter, which they call speckle reducing anisotropic diffusion (SRAD). This filter has shown very good performance with different levels of speckle. However, SRAD tends to broaden thin linear features and point features. These features carry useful information for diagnosis and the problem need to be corrected.

To overcome the feature broadening problem, a method has been proposed by Acton [9]. This method combines the strength of SRAD and deconvolution restoration. This technique assumed that feature distortion is caused by the convolution of the point spread function of the imaging system with the underlying feature. Hence, deconvolution sharpens features, while SRAD removes the speckle. This method showed promising results on synthesized US data, although no results were reported for real data. A generic framework to find the matrix-valued counterparts of the Perona-Malik PDE with various diffusivity functions is proposed by Burgeth et al. [10].

Fourth-order partial differential equation (PDE)-based despeckling method has been proposed in [11]. This can reduces the speckle and also able to keep the image edge better, but this method requires more number of iterations to converge.

Regularization methods have been used in real-valued image restoration [12,13], as well as image reconstruction problems such as medical tomography $[14,15]$ to obtain improved image estimates in the face of data degradation. The simplest and the most common approach is to use quadratic functions of the unknown quantities. These methods lead to computationally straightforward optimization problems, but they suppress useful features in the resulting imagery, such as edges. Recently, considerable effort has been spent in designing alternative, non-quadratic constraints which preserve such features. Methods based on these non-quadratic constraints have successfully been used in edge-preserving regularization in image restoration [12] and computer-assisted tomography [13-16].

In this article, a new method has been proposed to reduce speckle in US images by incorporating a nonquadratic regularization into nonlinear coherent diffusion to preserve and enhance edges, local details, and to correct the feature broadening distortion. The proposed model carries two terms: the first is coherent diffusion term that reduces the speckle by nonlinear coherent diffusion, which utilizes the diffusion tensor derived from coordinate transformation. The second term is called regulator, which enhance the performance of coherent diffusion as well as it enables the correction of feature broadening distortion. Therefore, our model performs simultaneous speckle reduction, structure enhancement, and feature broadening correction with minimum computational cost.

\section{Background of diffusion}

The basic idea in the use of PDEs in image processing is to deform an image, a curve, or a surface in a PDE framework and to approach the expected result as a solution to this equation.

Let $I: \Omega \rightarrow \Re$ be a scalar-valued image (gray level image) with $\Omega \subset \mathfrak{R}^{p}$. Gradient of the image characterizes the difference in gray value. In Biomedical imaging, besides noise also edges result in a large gradient at fine scales. The direct approach to reduce variations in the image $I$ would be to reduce the gradient of the image globally.

Calculus of variations is a mathematical tool with the help of which we can find the extrema of functionals.

$$
\min _{I: \Omega \rightarrow \Re} E(I)=\int_{\Omega} F\left(x, y, I(x, y), I_{x}(x, y), I_{y}(x, y)\right) d \Omega
$$

Euler-Lagrange equation gives a necessary condition that must be verified by $I$ to reach minimum of $E(I)$. And also one can use gradient descent an iterative 
approach to reach such a minimum, starting from an initial image. Equation (1) can be reduced as

$$
E(I)=\int_{\Omega} F(x, y, I(x, y), \nabla I(x, y)) d \Omega
$$

where $I(\mathrm{x}, \mathrm{y})$ is $2 \mathrm{D}$ image by

$$
\begin{cases}\frac{\partial F}{\partial I}-\frac{\partial}{\partial x} \frac{\partial F}{\partial I_{x}}-\frac{\partial}{\partial y} \frac{\partial F}{\partial I_{y}} & \text { for }(x, y) \in \Omega, \\ \nabla I . n=0 & f o r(x, y) \in \partial \Omega\end{cases}
$$

where $\partial \Omega$ is boundary of $\Omega$ and $n$ is normal vector of boundary. Euler-Lagrange equation makes the link between PDEs evolution and gradient descent for continual minimization.

Diffusion is a physical process that equilibrates concentration differences without creating or destroying mass. This physical observation is mathematically expressed by Flick's law as

$$
j=-D . \nabla I
$$

This equation states that a concentration gradient $\nabla I$ causes a flux $j$ which aims to compensate for this gradient [17]. The relation between $\nabla I$ and $j$ is described by the diffusion tensor $D$, a positive definite symmetric matrix. The case where $j$ and $\nabla I$ are parallel is called isotropic. Then we may replace the diffusion tensor by a positive scalar-valued diffusivity g. In general, for anisotropic case, $j$ and $\nabla I$ are not parallel. Thus the diffusion only transports mass without destroying it or creating new mass. This observation is expressed by the continuity equation as

$$
\partial_{t} I=-\operatorname{div} j
$$

where $t$ denotes the time. If we plug in Flick's law into the continuity equation we end up with the diffusion equation

$$
\partial_{t} I=\operatorname{div}(D . \nabla I)
$$

This equation appears in many physical transport processes. In the context of heat transfer, it is called heat equation. In image processing, we may identify the concentration with the grey value at a certain location. If the diffusion tensor is constant over the whole image domain, one speaks of homogeneous diffusion whereas a space-dependent filtering is called inhomogeneous. Often the diffusion tensor is a function of the differential structure of the evolving image itself. Such a feedback leads to nonlinear diffusion filters. Diffusion which does not depend on the evolving image is called linear. Sometimes the computer vision literature deviates from the preceding notations, i.e., the homogeneous filtering is named isotropic and inhomogeneous blurring is called anisotropic, even if it uses a scalar-valued diffusivity instead of a diffusion tensor.

\section{Anisotropic diffusion}

A general expression of the $\mathrm{AD}$ equation can be written as

$$
\left\{\begin{array}{cc}
\frac{\partial I}{\partial t}= & \begin{array}{c}
I(x, 0)=I_{0} \\
\operatorname{div}(F)+\beta\left(I_{0}-I\right)
\end{array}
\end{array}\right.
$$

where $F$ is the diffusion and $\beta$ is a data attachment coefficient. If $\beta=0$, particular cases of this equation are (1) the heat diffusion equation $F=\nabla I$, which is equivalent to a Gaussian convolution. (2) The Perona and Malik equation [6] with $F=g(\nabla I) \nabla I$, where $g$ is diffusion function. This function has the effect of reducing the diffusion for "high" gradients, based on the threshold $\beta$ on the norm of the gradient. (3) The matrix diffusion proposed in [16] uses a diffusion matrix noted $D$ with a flux $F=D . \nabla I$. The matrix $D$ can be expressed in a diagonal form with eigenvectors $\left(v_{0} ; v_{1} ; v_{2}\right)$ and eigen values $\lambda_{0}$, $\lambda_{1}, \lambda_{2}$. Then the flux can be expressed as

$$
F=D . \nabla I=\sum_{i=0}^{2} \lambda_{i} I_{v_{i}} \nu_{i}
$$

where $I_{v_{i}}=\nabla I v_{i}$ is the first-order derivative of the intensity in the direction of $v_{i}$. In 1990, Perona and Malik [6] proposed a PDE-based diffusion method for denoising. Their work made a great influence in this field and the diffusion equation can be expressed as

$$
\frac{\partial I}{\partial t}=\nabla \cdot[c(q) \cdot \nabla I]
$$

With initial condition: $I(x, y, 0)=I_{0}, I(x, y, 0) ; \mathfrak{R}^{2}=$ $\mathfrak{R}^{+}$is an image in the continuous domain, where $(x, y)$ specifies the spatial position; $t$ is an artificial time parameter; $c$ is the diffusion constant, and $\nabla I$ is the image gradient. The $c$ value is suggested to provide backward diffusion around intensity transitions and forward diffusion in smooth areas in favor of edge sharpening and noise removal. Edges and local details are the most interesting parts in diagnostic imaging for clinicians. Therefore, enhancement and preservation of edges and local details on denoising are very important. In Equation (9), $c$ is a scalar function and $\nabla I$ serves only as an edge detector rather than providing smoothing.

\section{Nonlinear coherent diffusion}

The diffusion coefficient is represented in tensor form by Abd-Elmoniem et.al. [18] to measure local coherence of structures. This made the diffusion process more directional in both the gradient and the contour directions as an alternative of scalar parameter-based diffusion coefficient [6-15]. Hence, the coherent diffusion model takes 
the form $\partial I(x, y, t) / \partial t=\operatorname{div}(D \nabla I)$, where $D \in \mathfrak{R}^{2 X 2}$ is a symmetric positive semi-definite diffusion tensor representing the required diffusion in both gradient and contour directions. There are two tensors widely used to detect the local coherence, namely, the structure tensor (also called scatter matrix or windowed second moment tensor) and the Hessian tensor, which represents the second-order derivatives. These can be expressed as follows

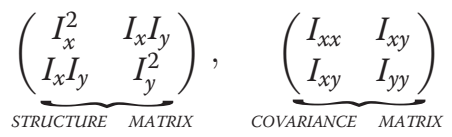

The Hessian matrix is more sensitive to noise; therefore, the structure tensor is favored. The following diffusion tensor is proposed in nonlinear coherent diffusion (NCD) model [18] to obtain selective smoothing along the gradient and contour direction.

$$
J(I)=\left(\begin{array}{ll}
\omega_{1} & \omega_{2}
\end{array}\right)\left(\begin{array}{cc}
\mu_{1} & 0 \\
0 & \mu_{2}
\end{array}\right)\left(\begin{array}{c}
\omega_{1}^{T} \\
\omega_{2}^{T}
\end{array}\right)
$$

where the eigen vectors $\omega_{1}$ and $\omega_{2}$ represent the directions of maximum and minimum variations and the eigen values $\mu_{1}$ and $\mu_{2}$ correspond to the strength of these variations, respectively. However, the diffusion tensor used in the nonlinear coherent diffusion model was actually depending on local statistics which are isotropic in nature, and also on the tensor provided by Gaussian smoothed image which may not effectively suppress the spatially correlated speckle noise.

\section{Proposed technique}

\section{Condensed anisotropic diffusion}

An image $I$ can be anisotropically smoothed, i.e., denoised with preservation of discontinuities by minimizing

$$
E_{\text {diff }}(I)=\int_{\Omega}\left[\frac{\alpha}{2} I-I_{0}^{2}+\phi(\nabla I)\right] d \Omega
$$

where is vector gradient norm that measures norm and orientation, i.e., global vector variation $\alpha \Re$ is fixed parameter that prevents the final solution at convergence to be too different from the original image. The function $\phi$ : $\Re \rightarrow \Re$ is a diffusion function that controls the regularization behavior. To minimize the functional $E_{\text {diff }}$ $(I)$, calculate the corresponding vector Lagrangian $L\left(E_{\mathrm{diff}}(I)\right) \in \mathfrak{R}^{n}$

$$
\nabla I=\sqrt{\sum_{i=1}^{n} \nabla I_{i}^{2}}
$$

By using a vector gradient descent: $\frac{\partial I}{\partial t}=-L\left(E_{\text {diff }}(I)\right)$;

$$
\left\{\begin{array}{l}
I(t=0)=I_{0} \\
\frac{\partial I_{i}}{\partial t}=\alpha\left(I_{i 0}-I_{i}\right)+\operatorname{div}\left(\frac{\phi^{\prime}(\nabla I)}{\nabla I} \nabla I_{i}\right)
\end{array}\right.
$$

For many years, image regularization with discontinuities (edges) preservation has been studied in the computer vision community. Image regularization with PDE is again based on a measure of local parameter variations. In Equation (14), the first term is regularization term $\alpha$ coupled with data attachment or fidelity term $\left(I_{i 0}-I_{i}\right)$.

By introducing orthonormal constraints in the minimization functionals equation (12) leads to the unconstrained minimization of,

$$
E(I)=E_{0}(I)+\beta \int_{\Omega}\left(I / I_{c}\right)^{\gamma} d \vec{x}
$$

The solution of (15) in the space of equal energy function is restricted to $\int_{\Omega} I^{\gamma} d \vec{x}=\int_{\Omega} I_{0}^{\gamma} d \vec{x}$ in order to avoid trivial solution of all zeros. The condensed anisotropic diffusion (CAD) model is derived by minimizing the energy function equations (14) and (15) as

$$
\frac{\partial I(x, y, t)}{\partial t}=\nabla \cdot(D \cdot \nabla I)+\beta \gamma\left(I_{c}\right)^{\gamma} I^{\gamma-1}(\vec{x}, t)
$$

To make the diffusion process more directional in gradient and contour direction, the diffusion coefficient can be put in tensor form $[18,19]$. And the tensor should be chosen such that to make the model progress from isotropic diffusion to AD. An isotropic diffusion is required in homogeneous domain and $\mathrm{AD}$ is needed in the domains of edges and local details. This means diffusion do occur only along the tangent direction of edges and does not across the edges. Diffusion tensor or covariance matrix $D$ is symmetric and semi-positive definite $n \times n$ matrix

$$
\begin{aligned}
& \text { Semi Positivity : } \forall k \in[1, n], \quad \lambda_{k} \geq 0, \\
& \text { Orthogonality (RealESymmetric) }: \forall k, l \in[1, n], \\
& u_{k, l}=\delta_{k l}, \\
& \delta_{k l}= \begin{cases}1, & \text { if } k=l \\
0, & \text { if } k \neq l\end{cases}
\end{aligned}
$$

$D$ can be expressed with eigen values $\lambda_{k}$ and its corresponding eigen vectors $u_{k}$. The meaningful information contained in $D$ can be retrieved from its spectral decomposition

$$
D=\sum_{k=1}^{n} \lambda_{k} u_{k} u_{k}^{T}=U A U^{T}
$$

$U=\left(u_{1}, u_{2}, \ldots, u_{n}\right)$ is the $n \times n$ orthogonal matrix of the unit eigen vector columns $u_{k}$, forming an orthonormal 
vector basis. $A=\operatorname{diag}\left(\lambda_{1}, \lambda_{2}, \ldots, \lambda_{n}\right)$ is the corresponding diagonal matrix of the positive eigen vectors. The spectral decomposition separates the orientation features and diffusivity features $A$ of tensor $D$.

For instance, to remove the noise effectively and to avoid the vision of undesired image structures, isotropic smoothing is preferred in homogeneous regions. Followed by, in order to preserve the vector edges while removing the noise, the diffusion should occur along the vector edges. And diffusion rate should be decreased in order to prevent the corner erosion. These conditions can be satisfied through the use of local coordinate transformation in the design of diffusion tensor. As shown in Figure 2, at the edge point $\boldsymbol{o}$, for $t \perp n, \vec{n}, \vec{t}$ are unit normal and unit tangent vectors, $\vec{n}=\frac{\nabla I}{|\nabla I|}, \vec{t}=\frac{\nabla I^{\perp}}{\mid \nabla I}$.

Where $t$ is tangent to the contours in the image everywhere and the set $(\vec{n}, \vec{t})$ is a moving orthonormal basis \& configuration depends on the current coordinate $(x, y)$. Using local coordinate transformation, the relationship between $(\vec{n}, \vec{t})$ and $(x, y)$ is given as

$$
\left(\begin{array}{c}
n \\
t
\end{array}\right)=\frac{1}{|\nabla I|} R_{\theta}\left(\begin{array}{l}
x \\
y
\end{array}\right)
$$

where $R_{\theta}$ is rotation matrix, for 2D image Equation (18) can be written as

$$
\left(\begin{array}{c}
\partial n \\
\partial t
\end{array}\right)=\frac{1}{|\nabla I|}\left(\begin{array}{cc}
I_{x} & I_{y} \\
-I_{y} & I_{x}
\end{array}\right)^{T}\left(\begin{array}{l}
\partial x \\
\partial y
\end{array}\right)
$$

From local coordinate transformation, the diffusion tensor becomes

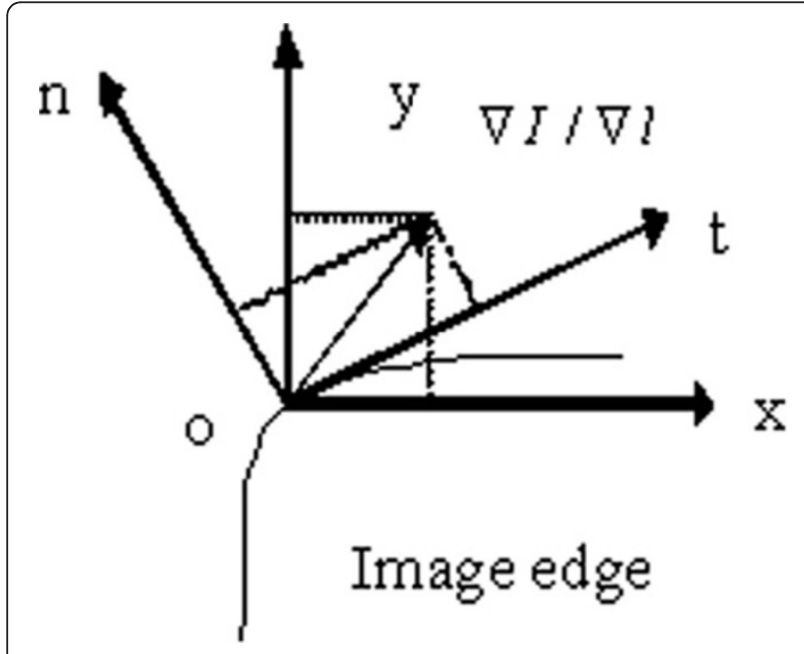

Figure 2 Decomposition of directional derivative on image edge.

$$
D=\frac{1}{|\nabla I|^{2}}\left(\begin{array}{cc}
I_{x} & I_{y} \\
-I_{y} & I_{x}
\end{array}\right)^{T}\left(\begin{array}{cc}
\lambda_{1} & 0 \\
0 & \lambda_{2}
\end{array}\right)\left(\begin{array}{cc}
I_{x} & I_{y} \\
-I_{y} & I_{x}
\end{array}\right)
$$

where $\lambda_{1}$ and $\lambda_{2}$ are diffusion coefficients along the direction of normal and tangent directions. In the NCD model [18], $\lambda_{1}$ and $\lambda_{2}$ are related to eigen value correspond to strength of maximum and minimum variations.

$$
\lambda_{1}=\left\{\begin{array}{cc}
\alpha\left(1-\left(\frac{A}{s}\right)^{2}\right), & \text { if } A=\left(\mu_{1}-\mu_{2}\right) \leq s^{2}, \lambda_{2}=\alpha \\
0, & \text { else }
\end{array}\right.
$$

In the constant regions, $\mu_{1} \approx \mu_{2} \approx 0$ and $\lambda_{1} \approx \lambda_{2} \approx \alpha$, which gives $D \approx \alpha I_{d}$ where, $I_{d}$ is identity matrix. Thus, in the constant (homogeneous) region, there is no preferred diffusion direction and the diffusion tensor is isotropic. For image contours, $\mu_{1}>>\mu_{2}>>0$ and $\lambda_{2}>\lambda_{1}>0$, diffusion tensor is anisotropic and mainly directed by the tangent vector of the image.

In (21), $\lambda_{1}$ is related to anisotropy of the image, i.e., $A=$ $\mu_{1}-\mu_{2}$, which is a monotonically decreasing function and resembles Tukey's bi-weight robust estimator. This function preserves sharp boundaries and improves automatic stopping of diffusion in the gradient direction. $s^{2}$ is the stopping level measured from the imaging system with respect to fully structured region and can be manually set. To avoid the energy loss in the image boundary during the diffusion process, the Neumann boundary condition is applied to the image border

$$
\begin{aligned}
& I(x, y, 0)=I_{0}, \\
& \partial_{n} I=0, \\
& \text { and } I_{c}=\operatorname{median}(I)
\end{aligned}
$$

The proposed CAD model is composed of two components: the nonlinear coherent diffusion component and the energy condensation component. The former accounts for speckle removal and the latter reduces the broadening distortion of point and linear features. According to the number of scatterers per resolution cell, the nature of speckle pattern is classified into three categories: Fully formed speckle (FFS) pattern [20], nonrandomly distributed with long range order [21,22], and non-randomly distributed with short range order [23]. The region corresponds to FFS carries less tissue information, i.e., small gradient variations and the diffusion must become isotropic along all directions, i.e., $\lambda_{1} \approx \lambda_{2}$. This condition can be accomplished by setting the local coherence measured by $\mu_{1}-\mu_{2}$ close to zero. On the other hand, the areas of edges and local details corresponding to structured tissue carries rich information about the imaged texture, i.e., big gradient variations. 
Therefore, the $\mathrm{AD}$ is needed in domains of edges and local details, which diffuse along the tangent direction of edges and not across the edges. In Equation (21), $\lambda_{1}$ is related to big gradient variation through Tukey's bi-weight robust estimator [18] and $\left(\lambda_{1}-\lambda_{2}\right)>s^{2}$ is related to fully structured region and diffusion occurs only in contour direction that is along $t$. The stopping level $s^{2}$ can be set manually.

To emphasize thin linear and point features in US image, which bear useful information for diagnosis, an energy condensation component is included in the proposed model. In Equation (16), $I_{c}$ is threshold value, which is set as mean of the image function $I(x, y)$. The weight factor $\beta$ is positive and it determines the amount of speckle smoothing, point and linear feature preservation. With $\gamma<<1$, the proposed condenser performs the following operation: First, the bright regions correspond to $I \geq I_{c}$ gets fat during the diffusion process and increases the total energy rapidly. Second, the majorities of darker regions corresponding to $I<I_{c}$, undergo NCD as $\left(\frac{I}{I_{c}}\right)^{\gamma} \rightarrow 0$. Thus, the condenser prevents the fattening of bright and linear structures without affecting the diffusion performed by the first term in Equation (16).

In the implementation of CAD model $\alpha=1, \mathrm{~s}=$ $70, \beta=0.05, \gamma=0.75, I_{c}=\langle I\rangle$ are chosen. After the iteration, the energy of the updated $I$ is rescaled by a factor of $\frac{\left\langle I_{0}\right\rangle}{\left\langle I_{0}\right\rangle}$, where \langle\rangle is mean value. Thus, the processed image has same energy as the input $I_{0}$.

\section{Discretization scheme}

The CAD is numerically implemented using finite difference scheme because of its easy implementation for $2 \mathrm{D}$ digital image. The CAD model can be expressed as

$$
\frac{\partial I(x, y, t)}{\partial t}=\nabla \cdot(D \cdot \nabla I)+\beta \gamma I_{c}^{-\gamma} I^{\gamma-1}(\vec{x}, t)
$$

In discrete form the first $\mathrm{AD}$ term can be expressed as

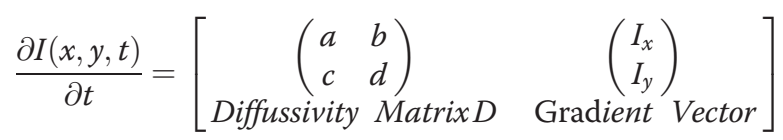

Using semi-implicit scheme, the CAD model can be expressed as

$$
\begin{aligned}
\frac{I_{i, j}^{n+1}-I_{i, j}^{n}}{\tau}= & D_{N}\left(\nabla_{N} I_{i, j}^{n}\right)+D_{S}\left(\nabla_{S} I_{i, j}^{n}\right)+D_{E}\left(\nabla_{E} I_{i, j}^{n}\right) \\
& +D_{W}\left(\nabla_{W} I_{i, j}^{n}\right)+\beta \gamma\left(I_{c i, j}^{n}\right)^{\gamma}\left(I_{i, j}^{n}\right)^{\gamma-1}
\end{aligned}
$$

where

$$
\begin{aligned}
& \nabla_{N} I_{i, j}^{n}=I_{i-1, j}^{n}-I_{i, j}^{n} \\
& \nabla_{S} I_{i, j}^{n}=I_{i+1, j}^{n}-I_{i, j}^{n} \\
& \nabla_{E} I_{i, j}^{n}=I_{i, j+1}^{n}-I_{i, j}^{n} \\
& \nabla_{W} I_{i, j}^{n}=I_{i, j-1}^{n}-I_{i, j}^{n}
\end{aligned}
$$

The spatial distance between two neighboring pixels is one, i.e., $h=1$ for image and $\tau$ is the time interval between the consecutive iterations. $I_{i, j}^{n}$ refers to present pixel value at location $(i, j)$ at time $n, I_{i, j}^{n+1}$ is the pixel value at the same location at time $n+1$. The diffusion coefficients $D_{N}, D_{S}, D_{E}, D_{W}$ are calculated from formulae (14) to (16). $I_{c i, j}^{n}$ is median of $I_{i, j}^{n}$. The $\gamma$ value is chosen empirically as 0.75 (for $\gamma<<1, \beta=0$ ). After every iteration the energy is rescaled by a factor of $\frac{\left\langle I_{0}\right\rangle}{\langle I\rangle}$, where \langle\rangle denotes the mean value. Therefore, the processed image remains in the same energy as the input $I_{0}$. The time step is set to 0.25 such that $0 \leq \gamma \leq \frac{h^{2}}{4}$. As a result,

$$
I_{i, j}^{n+1}=I_{i, j}^{n}+\frac{D_{N}\left(\nabla_{N} I_{i, j}^{n}\right)+D_{S}\left(\nabla_{S} I_{i, j}^{n}\right)+D_{E}\left(\nabla_{E} I_{i, j}^{n}\right)+D_{W}\left(\nabla_{W} I_{i, j}^{n}\right)}{+\beta \gamma\left(I_{c i, j}^{n}\right)^{\gamma}\left(I_{i, j}^{n}\right)^{\gamma}-1}
$$

When $\beta=0$, the image region undergoes nonlinear coherent diffusion. The $\beta$ value should be chosen such that it prevents the fattening of bright structures without affecting normal non linear coherent diffusion in dominant image regions. The technique in [20] can be used to find the value of $\beta$.

As the diffusion process is iterative, the important task lies in deciding the stopping criteria. Several automatic optimal stopping time estimation criteria are available in the literature [24-26]. In our simulation, we preferred the mean absolute error (MAE) between two adjacent steps [27] to stop the iterations.

$$
M A E=\frac{1}{M N} \sum_{i=1}^{M} \sum_{j=1}^{N}\left|\left(I_{i, j}^{n}-I_{i, j}^{n-1}\right)\right|
$$

where $M, N$ are number of columns and rows in the processed image. By setting a threshold for MAE value, the diffusion process can be stopped. This threshold value can be adjusted by clinicians according to the purpose of speckle reduction. When the despeckling method is used as a visual aid to improve the interpretation, a small diffusion time is enough to remove the speckle. On the other hand, if the method is applied as a preprocessing step, a longer diffusion time can be adopted. 


\section{Proposed algorithm}

An iteration $k$ of the proposed algorithm consists of the following steps:

Step 1: For each point $(x, y)$ belongs to 2D space of all real numbers $(x, y) \in \mathfrak{R}^{2}$, calculate the gradient in $x$ and $y$ directions and estimate the absolute gradient magnitude $|\nabla I|=\sqrt{I_{x}^{2}+I_{y}^{2}}$ for local window of size $w \times w$.

Step 2: Evaluate the diffusivity from Equation (20) and the principal components from Equations (21) and (11).

Step 3: Calculate the median of $I$ for each coordinate.

Step 4: Solve the diffusion equation in (27) to update $I_{i, j}^{n+1}$ from $I_{i, j}^{n}$ and the calculated matrices at step $n$ using the semi implicit scheme.

Step 5: Complete all the pixels in the image and check for stopping criteria as in (28) as a function of $n$. Loop until the stopping a criterion is satisfied for time step $\tau=0.25$.

\section{Experiments and results}

The performance of the proposed method is evaluated using artificial image, simulated phantom, and real US image. In each study, the performance of the proposed CAD is compared with Perona and Malik diffusion (PM), adaptive weighted median filter (AWMF) [28], SRAD [8], nonlinear coherent diffusion (NCD) [18], median boosted anisotropic diffusion (MBAD) [29], and Laplacian pyramidbased nonlinear diffusion (LPND) [27].

The performance of our method is quantified using quality assessment metrics. The edge preservation ability is measured using figure-of-merit (FOM) [30,31] and is based on three things: detection, localization, and spurious response.

$$
F O M=\frac{1}{\max \left\{\hat{N}, N_{\text {ideal }}\right\}} \sum_{i=1}^{N} \frac{1}{1+d_{i}^{2} \lambda}
$$

where $N$ and $N_{\text {ideal }}$ are the numbers of detected and original edge pixels, respectively; $d_{i}$ is the Euclidean distance between the $i$ th is a constant typically set to $1 / 9$. Dynamic range of detected edge pixel and the nearest original edge pixel; $\lambda$ FOM is based on all edges being found, all being placed in the correct location and no false alarms. The value is between the processed image and the ideal image. We used the canny edge detector [32] to find the edge in all processed results.

Second metric is mean square error in this metric, the smaller the MSE value, the better is the denoising process. The convergence rate of MSE value is depicted in Figure 3 for CAD algorithm with respect to number of iterations.

$$
M S E=\frac{1}{M N} \sum_{i=1}^{M} \sum_{j=1}^{N}\left(I_{\text {original }}(i, j)-I_{\text {denoised }}(i, j)\right)^{2}
$$

Third, the quality of the image is measured using signal-to-noise ratio (SNR) [32]

$$
\mathrm{SNR}=10 \cdot \log _{10}\left(\frac{\sigma_{g}^{2}}{\sigma_{e}^{2}}\right)
$$

where $\sigma_{g}^{2}, \sigma_{e}^{2}$ are the variances of the noise free reference image, the error between the original and denoised image, respectively.

Fourth, the structural similarity index (SSIM) is preferred as a quality assessment factor [32] that characterizes the luminance, contrast, and structural changes,

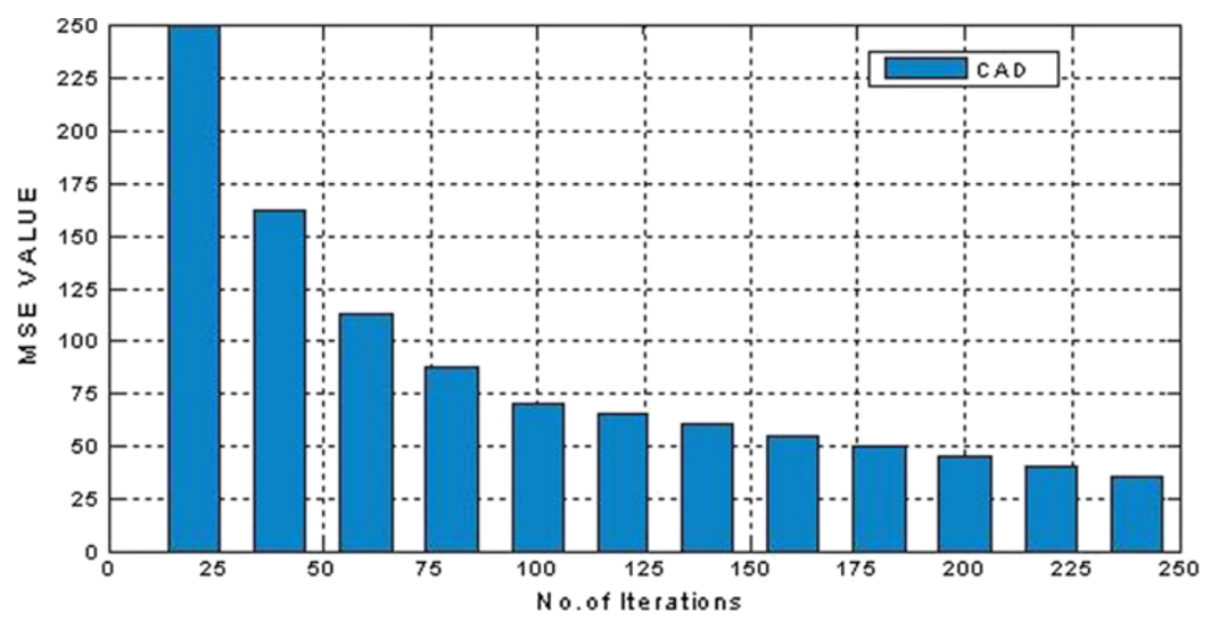

Figure 3 MSE value versus number of iterations. 
Table 1 Execution time of compared algorithms

\begin{tabular}{llc}
\hline & Number of iterations & Execution time (s) \\
\hline PM & 300 & 73.65 \\
AWMF & - & 62.95 \\
SRAD & 300 & 12.08 \\
NCD & 300 & 18.06 \\
MBAD & 300 & 26.82 \\
LPND & 300 & 20.68 \\
CAD & 300 & 10.34 \\
\hline
\end{tabular}

$$
\operatorname{SSIM}(x, y)=\frac{\left(2 \mu_{x} \mu_{y}+C_{1}\right)\left(2 \sigma_{x y}+C_{2}\right)}{\left(\mu_{x}^{2}+\mu_{y}^{2}+C_{1}\right)\left(\sigma_{x}^{2}+\sigma_{y}^{2}+C_{2}\right)}
$$

where the standard deviation $\sigma_{x}=\left(\frac{1}{N-1} \sum_{i=1}^{N}\left(x_{i}-\mu_{x}\right)^{2}\right)^{1 / 2}$ and the mean intensity $\mu_{x}=\frac{1}{N} \sum_{i=1}^{N} x_{i}$, covariance $\sigma_{x y}=$ $\frac{1}{N-1} \sum_{i=1}^{N}\left(x_{i}-\mu_{x}\right)\left(y_{i}-\mu_{y}\right)$ are calculated using local statistics within a total of $N$ windows. Constants $C_{1}, C_{2}$ $<<1$ to ensure stability and $N$ is chosen as 32 . The

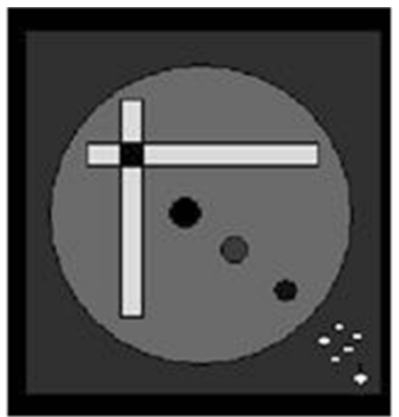

(a)

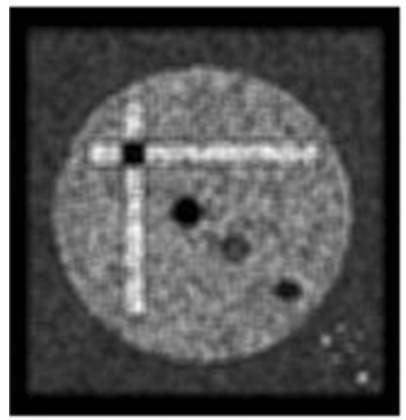

(d)

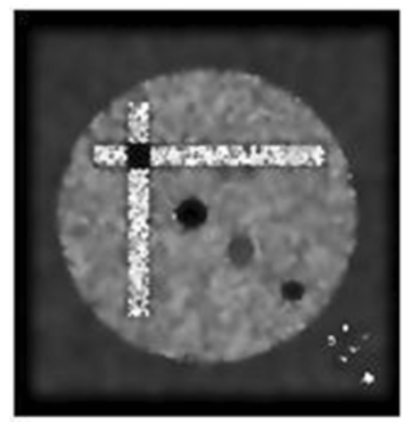

(g)

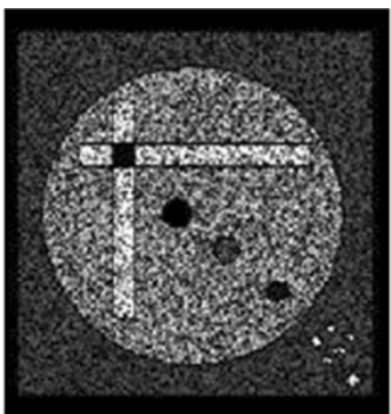

(b)

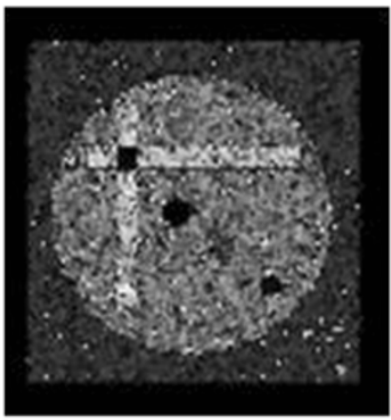

(e)

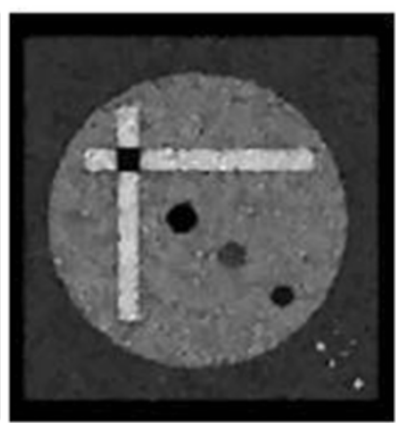

(h)

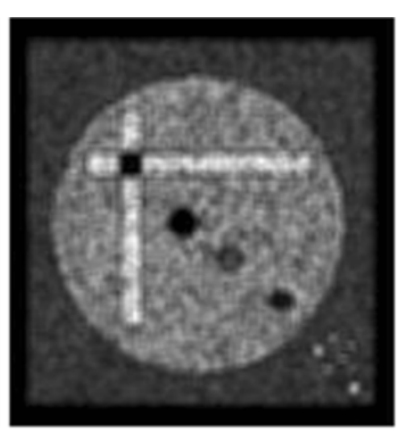

(c)

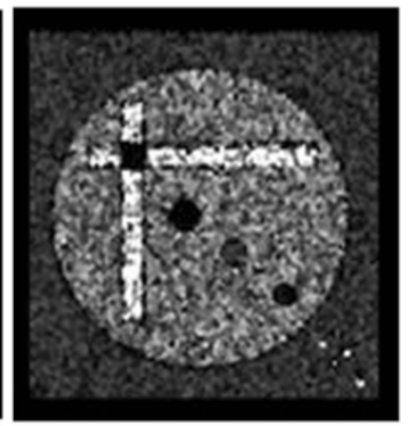

(f)

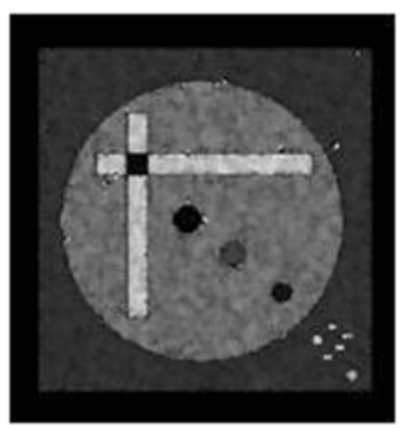

(i)

Figure 4 Simulated B mode image and its filtered results. (a) Echogeneity map. (b) Speckled image. (c- i) Images filtered by PM, AWMF, SRAD, NCD, MBAD, LPND, and CAD. 

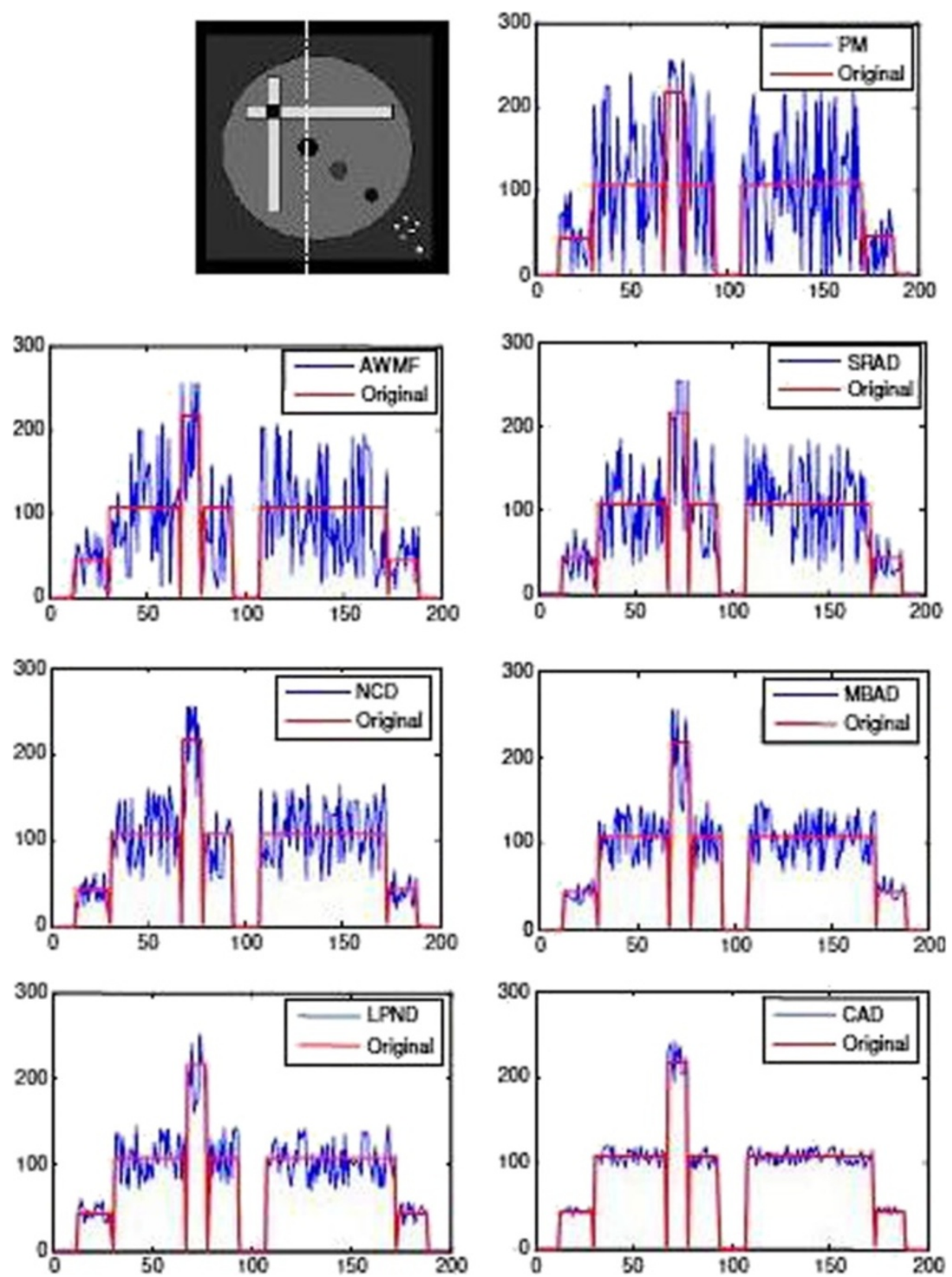

Figure 5 Image profile along 89th column of simulated B mode image.

SSIM has values in the 0 to 1 range, with unity representing structurally identical images. The SSIM values are calculated only for simulated images for which the original is available for comparison.

For real US data set a contrast image measure is estimated as

$$
C(x, y)=\frac{I_{\max }(x, y)-I_{\min }(x, y)}{I_{\max }(x, y)+I_{\min }(x, y)}
$$

In the above measure, $I_{\max }(x, y), I_{\min }(x, y)$ are the maximum and minimum values of the pixels in the $2 n+1$ selected neighborhood. The performance of CAD model can be measured by

$$
C=\frac{\sum_{x, y \in \Omega} C(x, y)}{N}
$$

where $\Omega$ is image region, $N$ is pixel no. in the region. For good diffusion model, the homogeneous region in the image exhibits less contrast after diffusion than compared to the original one. 
Table 2 Performance measures

\begin{tabular}{|c|c|c|c|c|c|c|c|c|}
\hline \multirow[t]{2}{*}{ Method } & \multicolumn{3}{|c|}{ Simulated B mode image } & \multicolumn{3}{|c|}{ Field II simulated foetus image } & \multicolumn{2}{|c|}{ Real US image } \\
\hline & $\overline{\text { SNR }}$ & MSE & FOM & $\overline{\text { SNR }}$ & MSE & FOM & SNR & FOM \\
\hline Noisy & 15.867 & 100.56 & 0.0969 & 25.538 & 121.69 & 0.0907 & 23.718 & 0.0976 \\
\hline PM & 19.486 & 88.64 & 0.1092 & 28.937 & 84.85 & 0.1762 & 25.645 & 0.1792 \\
\hline AWMF & 20.097 & 92.63 & 0.2206 & 29.427 & 91.02 & 0.2009 & 27.097 & 0.2461 \\
\hline SRAD & 24.468 & 51.84 & 0.3325 & 32.618 & 58.69 & 0.3786 & 29.678 & 0.3357 \\
\hline NCD & 28.093 & 76.92 & 0.3768 & 34.936 & 78.09 & 0.3868 & 31.493 & 0.3668 \\
\hline MBAD & 32.386 & 58.89 & 0.3976 & 35.227 & 62.56 & 0.3902 & 32.386 & 0.4462 \\
\hline LPND & 36.753 & 45.63 & 0.4186 & 38. 538 & 58.63 & 0.4409 & 37.789 & 0.4798 \\
\hline CAD & 41.923 & 16.246 & 0.5032 & 44.419 & 19.56 & 0.5156 & 44.087 & 0.5332 \\
\hline
\end{tabular}

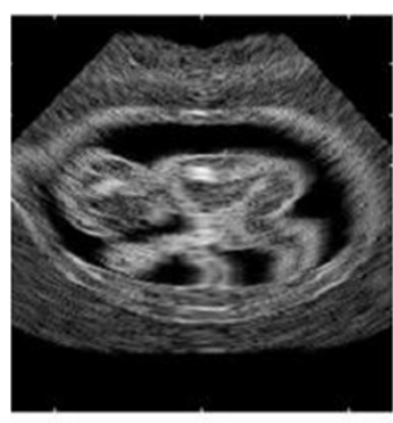

(a)

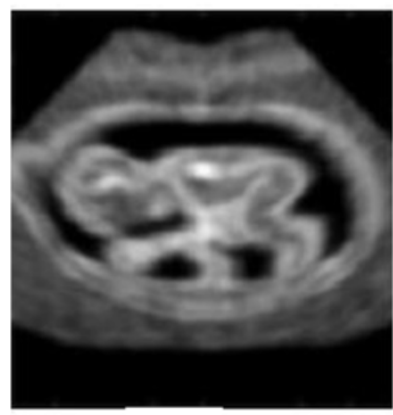

(d)

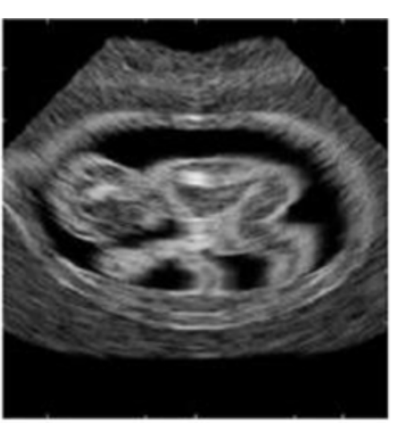

(b)

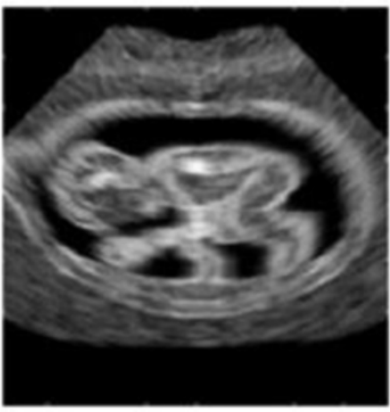

(e)

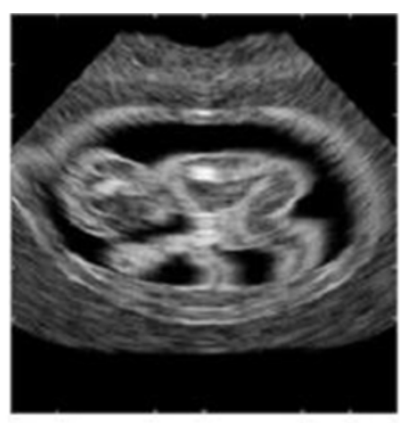

(c)

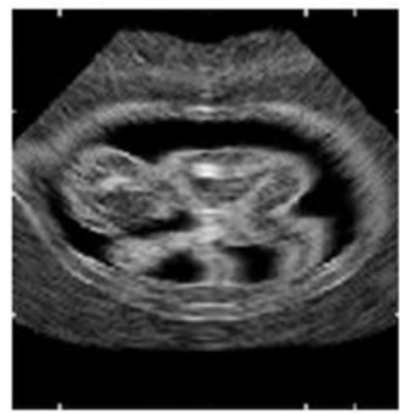

(f)

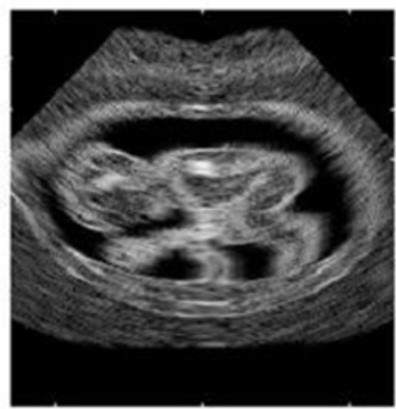

(g)

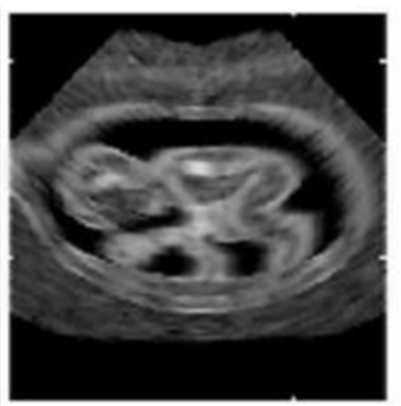

(h)

Figure 6 Simulated foetus image and its filtered results. (a) Noisy image. (b) Speckled image. (c-i) Images filtered by PM, AWMF, SRAD, NCD, $M B A D, L P N D$, and CAD. 

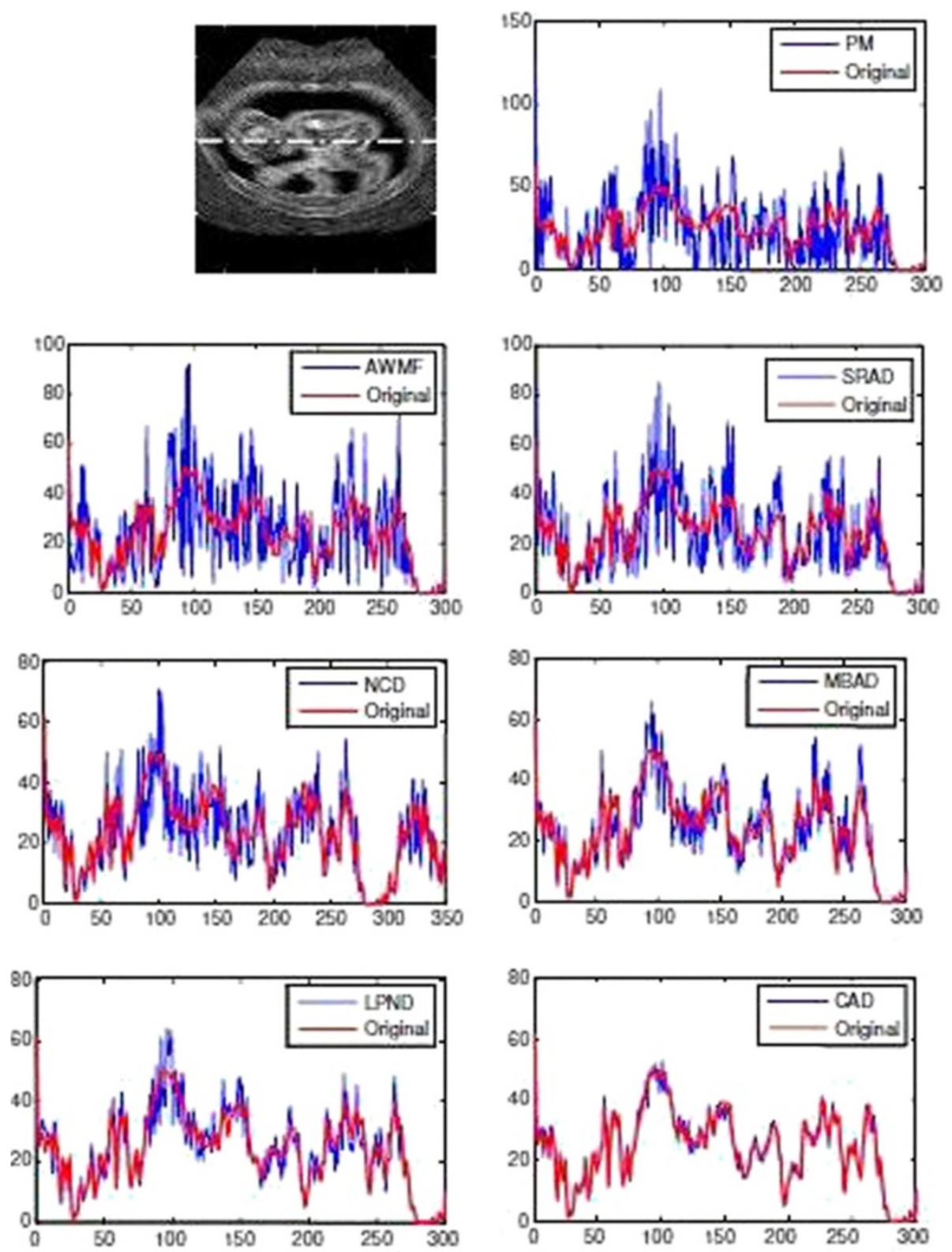

Figure 7 Image profile along 151 st row of simulated Field II foetus image.

The proposed CAD model and various state-of-the-art methods are applied on artificially simulated image with speckle noise of standard deviation 0.5, Field II software generated cyst and real US pediatric brain image. In this study, for PM and SRAD implementation, the time step $\Delta t=0.05$ is chosen and 300 iterations are used. For LPND, 4 pyramid layers, $7 \times 7$ binomial filter for $R E$ $D U C E$ and EXPAND operator, the Gaussian filter of $\sigma=$ $0.1, \Delta t=0.2$, and MAE $=0.1$ are set. For NCD $\Delta t=3, \alpha$ $=1, s=70$, and $\beta=0.05$ are selected for MBAD and AWMF, the filter mask of $5 \times 5$, median filter with $\sigma=2$ and $\Delta t=0.2$ are utilized. The execution time for each algorithm for 300 iterations is listed in Table 1.

The artificially simulated image carries intersected horizontal and vertical rectangular target, three small cysts of varying intensities and five point targets embedded in the background of varying gray levels. The results on artificial image are shown in Figure $4 \mathrm{a}-\mathrm{i}$. The original image of size $200 \times 200$ is artificially corrupted by speckle noise using MATLAB command ( $>>$ imnoise). The original and noisy images are shown in Figure $4 \mathrm{a}, \mathrm{b}$. The processed results of different schemes are shown in Figure $4 c-i$. In this 


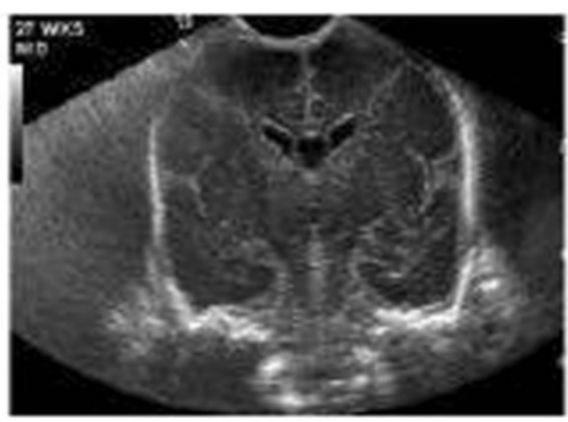

(a)

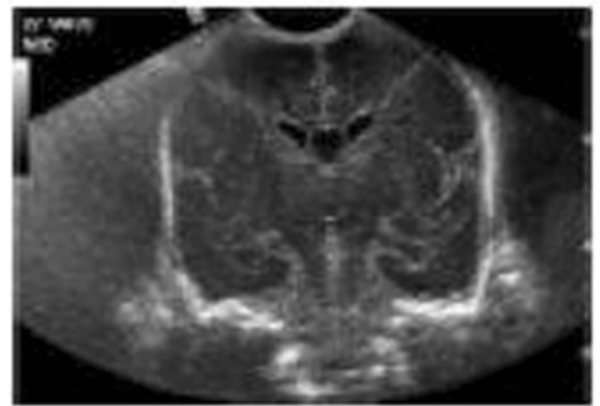

(c)

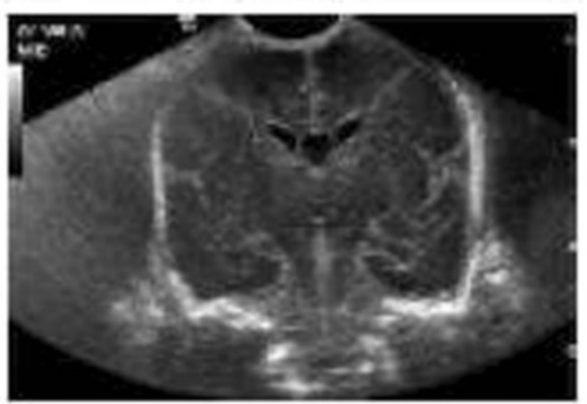

(e)

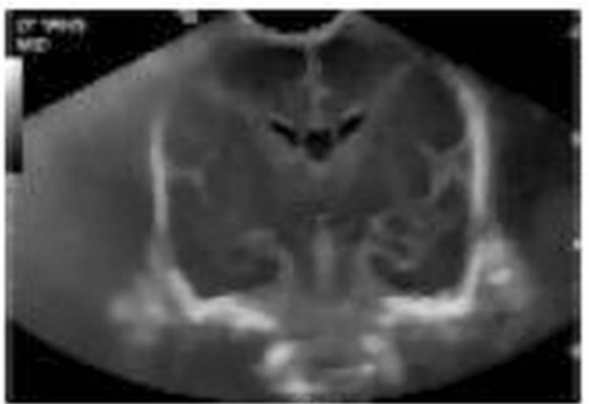

(g)

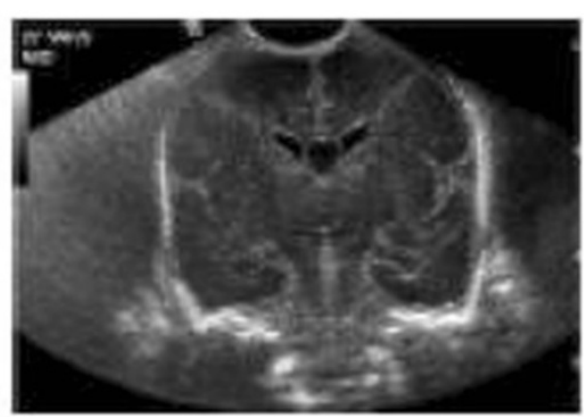

(b)

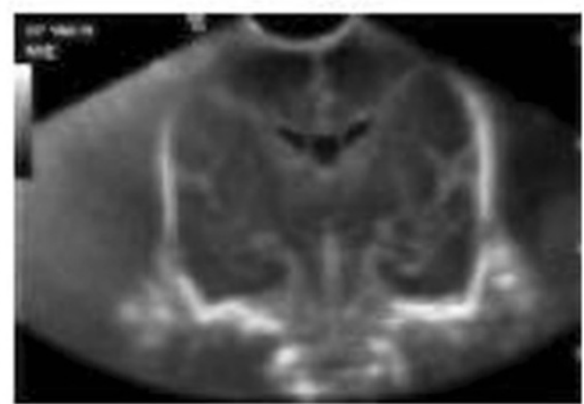

(d)

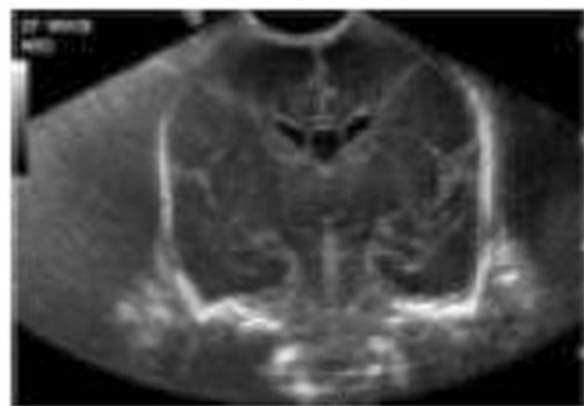

(f)

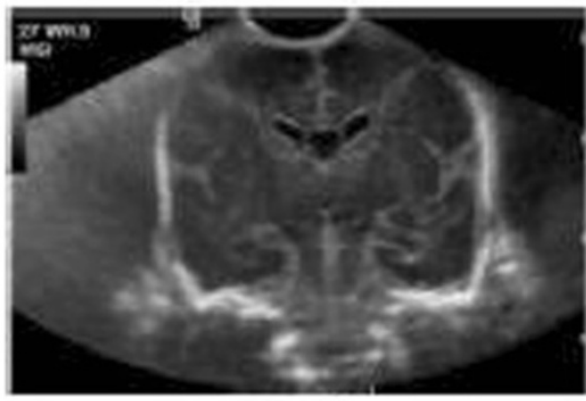

(h)

Figure 8 Real US pediatric image and its filtered results. (a) Noisy image. (b-h) Images filtered by PM, AWMF, SRAD, NCD, MBAD, LPND, and $C A D$.

example, our proposed CAD comparatively provides better visual enhancement, point feature preservation and also avoids blocky effects and feature broadening problem.
The performance of our method is also quantified using image profile measured along 89th column of the test image as shown in Figure 5. In each case, the profiles of 
filtered images are compared with profile of the original image. In this study, the CAD gives satisfied improvement over other techniques in terms of speckle reduction and edge enhancement. The three performance metrics SNR, FOM, and MSE are computed for all the methods and listed in Table 2. The CAD that carries larger SNR value corresponds to good quality. The FOM value indicates that the CAD is better than other methods in terms of edge preserving ability.

The performance of proposed method and other approaches on Field II software generated cyst are shown in Figure $6 \mathrm{a}-\mathrm{h}$. The foetus phantom is an 8-bit image of size $352 \times 353$ pixels. The image profile along 151st row of foetus image for CAD and other filtered results are shown in Figure $7 \mathrm{a}-\mathrm{h}$. The performance metrics were also calculated and listed in Table 2. All the experiments prove that our CAD gives improved result in terms of feature preservation, speckle reduction, and edge enhancement. An US image of pediatric brain of size $460 \times 312$ is used to test the performance of the proposed technique for real-time application. The results are displayed in Figure 8a-h.

This study depicts that LPND shows sharper but jagged edges and gives a relatively low contrast. AWMF

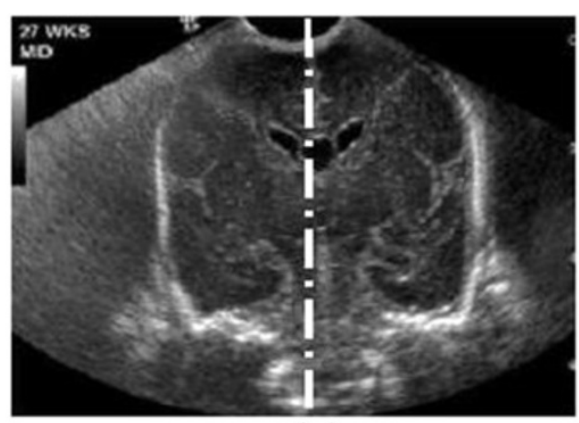

(a)

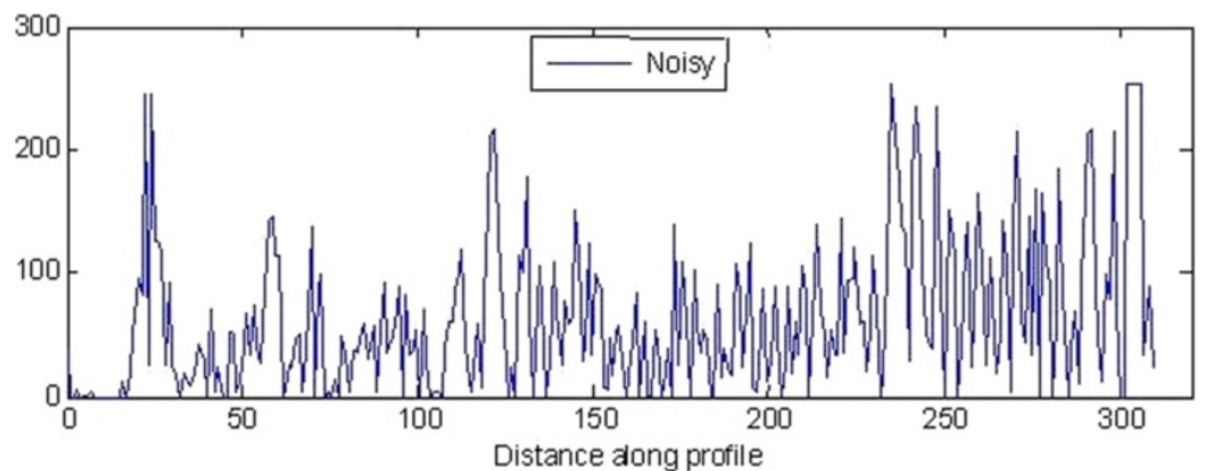

(b)

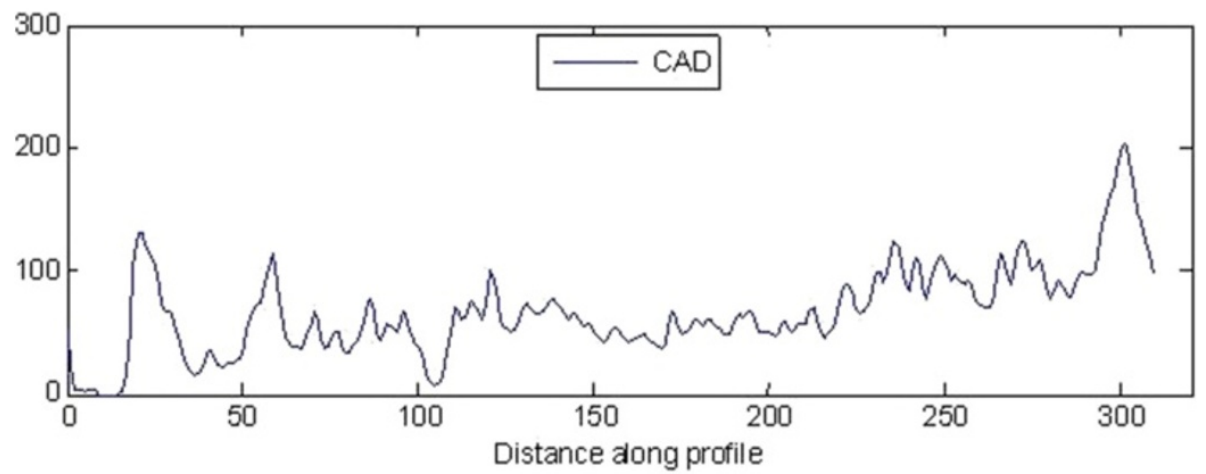

(c)

Figure 9 Image profile along 240th column of real US image. 
Table 3 SSIM value for the compared algorithms

\begin{tabular}{lcc}
\hline Method & \multicolumn{2}{l}{ SSIM value } \\
\cline { 2 - 3 } & Simulated B mode image & Field II simulated foetus image \\
\hline Noisy & 0.3512 & 0.2289 \\
PM & 0.6584 & 0.5709 \\
AWMF & 0.6987 & 0.5987 \\
SRAD & 0.7529 & 0.7297 \\
NCD & 0.8028 & 0.7832 \\
MBAD & 0.8163 & 0.8652 \\
LPND & 0.8209 & 0.8704 \\
CAD & 0.9567 & 0.9643 \\
\hline
\end{tabular}

does not give satisfactory speckle suppression. NCD enhances edges, but it does not keep correct edge locations. PM and SRAD dilate bright regions and erode dark regions. With the SRAD, the boundaries of bright regions are broadened and those of dark regions are shrunk. MBAD enhances the edge coherence but cannot suppress enough noise. The profile along 240th column of the real US image is shown in Figure 9. The image profile of original noisy image and filtered result of CAD are compared. The performance metrics for Field II simulated image and real US data are listed in Table 2. The experimental results show that our proposed CAD method provides better speckle reduction, edge enhancement, and feature preservation. It also avoids blocky effects and point/linear feature broadening problems.

SSIM values for different algorithms are calculated by using the constant values as referred in [32] for simulated phantom and Field II generated image for which the original images are accessible. The values are listed in Table 3. In this comparison, the proposed CAD exhibits comparatively high SSIM value for both the cases. For US image, the original noise-free image is not available and in this case, the contrast to noise ratio is calculated for two homogeneous regions in each case as shown in Figure 10. Contrast value of the homogeneous region should decrease after diffusion depending upon the quality of diffusion. Measured values of contrast for all three images for two different regions are listed in Table 3. In this study also our proposed CAD provides lower value of CNR compared to other state of the art algorithms (Table 4).
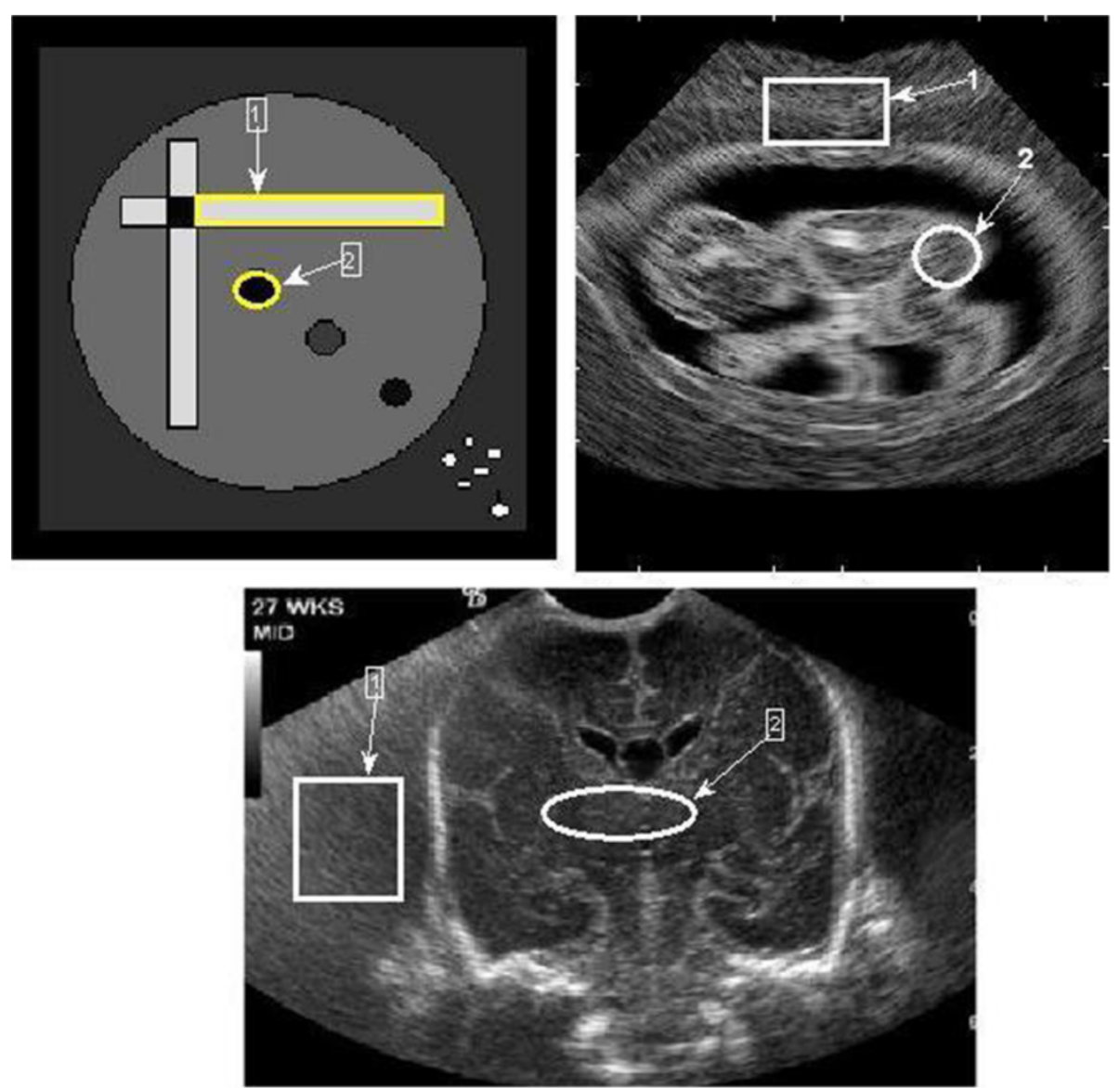

Figure 10 Simulated phantom, Field II generated foetus, and Real US image with two selected regions for contrast measure. 
Table 4 CNR value for the compared algorithms

\begin{tabular}{|c|c|c|c|c|c|c|}
\hline & \multicolumn{2}{|c|}{ Simulated B mode image } & \multicolumn{2}{|c|}{ Field II simulated foetus image } & \multicolumn{2}{|c|}{ Real US image } \\
\hline & Region 1 & Region 2 & Region 1 & Region 2 & Region 1 & Region 2 \\
\hline Original (Noisy) & 0.7862 & 0.7382 & 0.6489 & 0.6821 & 0.5656 & 0.5365 \\
\hline PM & 0.5289 & 0.4987 & 0.5332 & 0.5903 & 0.4087 & 0.4008 \\
\hline AWMF & 0.6032 & 0.5037 & 0.5123 & 0.5952 & 0.4239 & 0.4107 \\
\hline SRAD & 0.3185 & 0.3065 & 0.4165 & 0.4360 & 0.3543 & 0.3365 \\
\hline NCD & 0.2568 & 0.2167 & 0.3085 & 0.2987 & 0.2987 & 0.2754 \\
\hline MBAD & 0.2367 & 0.1754 & 0.2234 & 0.2145 & 0.1967 & 0.1965 \\
\hline LPND & 0.0938 & 0.0838 & 0.1045 & 0.1467 & 0.1245 & 0.1376 \\
\hline CAD & 0.0023 & 0.0021 & 0.0043 & 0.0035 & 0.0024 & 0.0032 \\
\hline
\end{tabular}
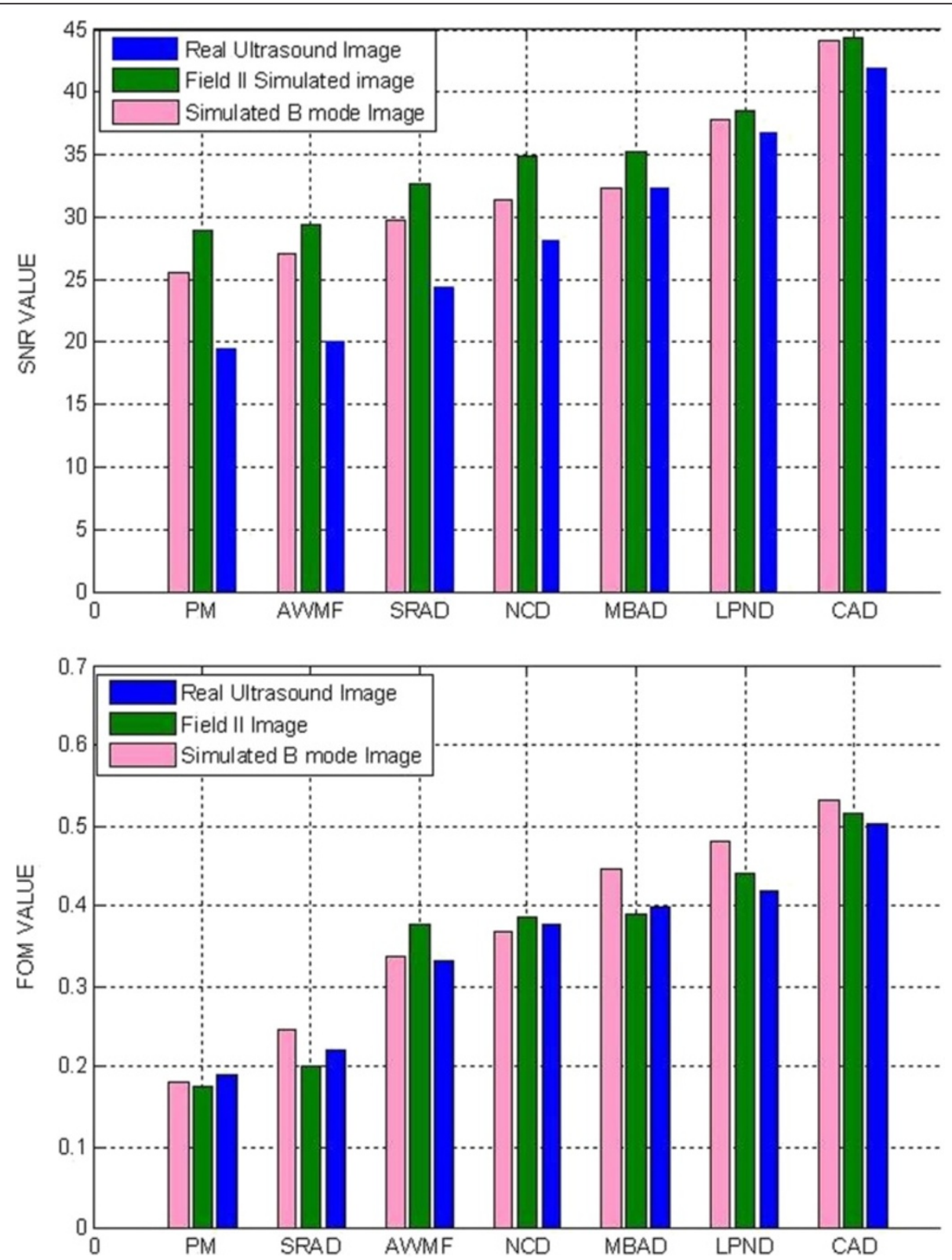

Figure 11 Performance comparisons of various methods in terms of (a) SNR (b) FOM. 
The performance of PM, AWMF, SRAD, NCD, MBAD, LPND, and CAD methods are compared in terms of SNR, SSIM, and FOM. Comparative results are shown in Figure 11 that clearly indicates that our proposed model gives superior result over the above-mentioned state-ofthe-art methods. In terms of SNR criterion, our proposed CAD model gives about 23, 22, 17, 14, 9, and $5 \mathrm{~dB}$ improvement over PM, AWMF, SRAD, NCD, MBAD, LPND, respectively, for artificially simulated image with speckle of $\sigma=0.5$, about $15,12,9$, and $5 \mathrm{~dB}$ improvement over PM, AWMF, SRAD, NCD, MBAD, LPND, respectively, for Field II simulated image, about $15,12,9$, and $6 \mathrm{~dB}$ improvement over PM, AWMF, SRAD, NCD, MBAD, and LPND, respectively, for real US image. Our proposed model gives $360,128,51,33.5,26.5$, and $20 \%$ improvement for artificially simulated image, $192,156,36,33,32$, and $17 \%$ improvement for Field II simulated image, 197, 117, 59, 45, 19.5, and $11 \%$ improvement for real US image over PM, AWMF, SRAD, NCD, MBAD, and LPND, respectively.

\section{Conclusions}

In this article, we propose a new diffusion model called CAD that reduces speckle, preserves information carrying features and also avoids blocking effects, point, and linear feature broadening problems. The new CAD model carries two terms: one is coherent diffusion term for speckle reduction and for structured region, organ surface preservation. The second term is a regulator term that condenses the diffusion and emphasizes thin linear and point features. In this scheme, the diffusion matrix is designed using local coordinate transformation and the feature broadening correction term is derived from energy function. The median filter is used as a smoothing operator. In CAD, the structured tissues which carry rich of information undergo $A D$ and the speckle pattern undergo isotropic diffusion; this flow can be controlled by setting the local coherence value close to zero. The energy condensation component is included to emphasize the information carrying point/linear features, which controls the feature fattening, effectively for bright regions. In the implementation, we have used. The MAE value is set to 0.1 for artificial and simulated data and 0.2 for real US image. The simulation takes $\approx 300$ iterations to converge to a stationary solution. Thus, the proposed method can be implemented practically to enhance the visual interpretation ability of radiologist with minimum cost and this method can also be used as a pre processing tool for many image processing task such as segmentation, feature extraction, etc

\section{Competing interest}

The authors declare that they have no competing interests.

\section{Acknowledgement}

The author would like to thank Dr. Senthilvelmurugan, Radiologist from Kauvery Medical Centre Hospital, Trichirappalli, Tamilnadu and Dr. G.
Jayakumar from Sarani clinic, Pudansandhai, Tamilnadu for their support and thoughtful comments.

\section{Author details}

${ }^{1}$ Department of Electronics and Communication Engineering, TRP Engineering College (SRM Group), Irungalur, Trichirappalli, Tamilnadu, India-621105. '2Department of Electronics and Communication Engineering, Government College of Engineering, Salem, Tamilnadu, India.

Received: 20 July 2011 Accepted: 18 June 2012

Published: 18 July 2012

\section{References}

1. R Touzi, A review of speckle filtering in the context of estimation theory. IEEE Trans. Geosci. Remote Sens. 40(11), 2392-2404 (2002)

2. J Lee, Digital image enhancement and noise filtering using local statistics. IEEE Trans. Pattern Anal. Mach. Intell. PAMI-2(2), 165-168 (1980)

3. V Frost, J Stiles, K Shanmugan, J Holzman, A model for radar images and its application to adaptive digital filtering of multiplicative noise. IEEE Trans. Pattern Anal. Mach. Intell. PAMI-4(2), 157-166 (1982)

4. DT Kuan, AA Sawchuk, T.C. Strand, P. Chavel, Adaptive noise smoothing filter for images with signal-dependent noise. IEEE Trans. Pattern Anal. Mach. Intell. PAMI-7(2), 165-177 (1985)

5. DT Kuan, AA Sawchuk, TC Strand, P Chavel, Adaptive restoration of images with speckle. IEEE Trans Acoust. Speech Signal Process. ASSP-35(3), 373-383 (1987)

6. P Perona, J Malik, Scale-space and edge detection using anisotropic diffusion. IEEE Trans. Pattern Anal. Mach. Intell. 12(7), 629-639 (1990)

7. J Monteil, A Beghdadi, A new interpretation and improvement of the nonlinear anisotropic diffusion for image enhancement. IEEE Trans. Pattern Anal. Mach. Intell. 21(9), 940-946 (1999)

8. Y Yu, T Acton, Speckle reducing anisotropic diffusion. IEEE Trans. Image Process. 11(11), 1260-1270 (2002)

9. ST Acton, Deconvolutional speckle reducing anisotropic diffusion. IEEE International Conference on Image Processing, ICIP 1, 5-8 (2005)

10. B Burgeth, S Didas, L Florack, J Weickert, A generic approach to diffusion filtering of matrix-fields. Computing 81, 179-197 (2007)

11. Y-L You, M Kaveh, Fourth-order partial differential equations for noise removal. IEEE Trans. Image Process. 9(10), 1723-1730 (2009)

12. MR Banham, AG Katsaggelos, Digital image restoration. IEEE Signal Process 14, 24-41 (1997). Mag

13. CR Vogel, ME Oman, Fast, robust total variation-based reconstruction of noisy, blurred images. IEEE Trans. Image Process 7, 813-824 (1998)

14. P Charbonnier, L Blanc-Féraud, G Aubert, M Barlaud, Deterministic edgepreserving regularization in computed imaging. IEEE Trans. Image Process 6 , 298-310 (1997)

15. P Coupe, P Hellier, C Kervrann, C Barillot, Nonlocal means-based speckle filtering for ultrasound images. IEEE Trans. Image Process. 18(10), 2221-2229 (2009)

16. AH Delaney, Y Bresler, Globally convergent edge-preserving regularized reconstruction: an application to limited-angle tomography. IEEE Trans. Image Process. 7, 204-221 (1998)

17. J Weickert, Anisotropic Diffusion in Image Processing (Teubner-Verlag, Stuttgart, 1998)

18. KZ Abd-Elmoniem, AM Youssef, YM Kadah, Real-time speckle reduction and coherence enhancement in ultrasound imaging via nonlinear anisotropic diffusion. IEEE Trans. Biomed. Eng. 49(9), 997-1014 (2002)

19. C Feddern, J Weickert, B Burgeth, M Welk, Curvature driven PDE methods for matrix valued images. Int. J. Comput. Vis. 69(1), 91-103 (2006)

20. RF Wagner, SW Smith, JM Sandrik, Statistics of speckle in ultrasound B-scans. IEEE Trans. Sonics Ultrason. 30(3), 156-163 (1983)

21. Karmeshu, R Agrawal, Study of ultrasonic echo envelope based on Nakagamiinverse Gaussian distribution. Ultrasound Med. Biol. 32, 371-376 (2003)

22. PM Shankar, Ultrasonic tissue characterization using a generalized Nakagami model. IEEE Trans. Ultrason. Ferroelectr. Freq. Control 48(4), 1716-1720 (2001)

23. T Eltoft, Modeling the amplitude statistics of ultrasonic images. IEEE Trans. Med. Imag. 25(2), 229-240 (2006)

24. MA Gennert, AL Yuille, Determining the optimal weights in multiple objective function optimization. International Conference on Computer Vision, 87-89 (1988)

25. J Weickert, Coherence-enhancing diffusion of colour images. Image Vis. Comput. 17(3), 201-212 (1999) 
26. P Mrázek, M Navara, Selection of optimal stopping time for nonlinear diffusion filtering. Int. J. Comput. Vis. 52, 189-203 (2003)

27. F Zhang, LM Koh, YM Yoo, Y Kim, Nonlinear diffusion in Laplacian pyramid domain for ultrasonic speckle reduction. IEEE Trans. Med. Imag. 26(2), 200-211 (2007)

28. T Loupas, WN McDicken, PL Allan, An adaptive weighted median filter for speckle suppression in medical ultrasound images. IEEE Trans. Circ. Syst. 36(1), 129-135 (1989)

29. Z Yang, MD Fox, Speckle reduction and structure enhancement by multichannel median boosted anisotropic diffusion. EURASIP J. Appl. Signal Process. 16, 2492-2502 (2004)

30. OV Michailovich, A. Tannenbaum, Despeckling of medical ultrasound images. IEEE Trans. Ultrason. Ferroelectr. Freq. Control 53(1), 64-78 (2006)

31. WK Pratt, Digital Image Processing (Wiley, New York, 1978)

32. Z Wang, AC Bovik, HR Sheikh, EP Simoncelli, Image quality assessment: from error visibility to structural similarity. IEEE Trans. Image Process. 13(4), 600-612 (2004)

doi:10.1186/1687-5281-2012-12

Cite this article as: Shanmugam and RSD: Condensed anisotropic diffusion for speckle reducton and enhancement in ultrasonography. EURASIP Journal on Image and Video Processing 2012 2012:12.

\section{Submit your manuscript to a SpringerOpen ${ }^{\circ}$ journal and benefit from:}

- Convenient online submission

- Rigorous peer review

- Immediate publication on acceptance

- Open access: articles freely available online

- High visibility within the field

- Retaining the copyright to your article 